\title{
Quotients of fake projective planes
}

\author{
JONGHAE KEUM
}

\begin{abstract}
Recently, Prasad and Yeung classified all possible fundamental groups of fake projective planes. According to their result, many fake projective planes admit a nontrivial group of automorphisms, and in that case it is isomorphic to $\mathbb{Z} / 3 \mathbb{Z}, \mathbb{Z} / 7 \mathbb{Z}, 7: 3$ or $(\mathbb{Z} / 3 \mathbb{Z})^{2}$, where $7: 3$ is the unique nonabelian group of order 21 .

Let $G$ be a group of automorphisms of a fake projective plane $X$. In this paper we classify all possible structures of the quotient surface $X / G$ and its minimal resolution.
\end{abstract}

$14 \mathrm{~J} 29 ; 14 \mathrm{~J} 27$

\section{Introduction}

It is known that a compact complex surface with the same Betti numbers as the complex projective plane $\mathbb{C} \mathbb{P}^{2}$ is projective (see eg Barth et al [2]). Such a surface is called $a$ fake projective plane if it is not isomorphic to $\mathbb{C P}^{2}$.

Mumford [12] first proved the existence of a fake projective plane, based on the theory of the $p$-adic unit ball by Kurihara [10] and Mustafin [13]. Later, using a similar idea, Ishida and Kato [6] proved the existence of at least two more. Then, Keum [8] gave a construction of a fake projective plane with an order 7 automorphism, using Ishida's description [5] of an elliptic surface covered by a (blow-up) of Mumford's fake projective plane. Recently, Prasad and Yeung [16] classified all possible fundamental groups of fake projective planes. According to their result, Keum's fake projective plane and Mumford's fake projective plane are different from each other, but belong to the same class. Furthermore, a group of automorphisms of a fake projective plane is isomorphic to $\{1\}, \mathbb{Z} / 3 \mathbb{Z}, \mathbb{Z} / 7 \mathbb{Z}, 7: 3$ or $(\mathbb{Z} / 3 \mathbb{Z})^{2}$, and many fake projective planes admit a nontrivial automorphism. Here $7: 3=(\mathbb{Z} / 7 \mathbb{Z}) \rtimes(\mathbb{Z} / 3 \mathbb{Z})$ is the unique nonabelian group of order 21 .

Let $G$ be a group of automorphisms of a fake projective plane $X$. In this paper we classify all possible structures of the quotient surface $X / G$ and its minimal resolution. We first deal with the case where $G$ is of prime order and prove the following: 
Theorem 1.1 Let $G$ be a group of automorphisms of a fake projective plane $X$. Let $Z=X / G$ and $v: Y \rightarrow Z$ be a minimal resolution. Then the following two statements are true.

(1) If the order of $G$ is 3 , then $Z$ has 3 singular points of type $\frac{1}{3}(1,2)$, and $Y$ is a minimal surface of general type with $K_{Y}^{2}=3, p_{g}=0$.

(2) If the order of $G$ is 7 , then $Z$ has 3 singular points of type $\frac{1}{7}(1,3)$, and $Y$ is a minimal elliptic surface of Kodaira dimension 1 with 2 multiple fibres. The pair of the multiplicities is one of the following three cases: $(2,3),(2,4),(3,3)$.

We remark that the fundamental group $\pi_{1}(Y)$ of $Y$ is given by $\{1\}, \mathbb{Z} / 2 \mathbb{Z}, \mathbb{Z} / 3 \mathbb{Z}$ in the three cases of (2), respectively. (See Dolgachev [3] for fundamental groups of elliptic surfaces.) The first case of (2), where $Y$ is called a Dolgachev surface, is supported by the example from Keum [8]. I have learnt from Donald Cartwright and Tim Steger that according to their computer calculation, there are examples of an order 7 quotient $Z$ of a fake projective plane with $\pi_{1}(Z)$ either $\{1\}$ or $\mathbb{Z} / 2 \mathbb{Z}$. This implies that the second case of (2) is supported by an example. We do not know if the third case of (2) actually occurs.

Corollary 1.2 Let $X$ be a fake projective plane with $\operatorname{Aut}(X) \cong(\mathbb{Z} / 3 \mathbb{Z})^{2}$. Let $G=$ $\operatorname{Aut}(X), Z=X / G$ and $v: Y \rightarrow Z$ be a minimal resolution. Then $Z$ has 4 singular points of type $\frac{1}{3}(1,2)$, and $Y$ is a numerical Godeaux surface, ie a minimal surface of general type with $K_{Y}^{2}=1, p_{g}=0$.

The above corollary proves the existence of a numerical Godeaux surface with a configuration of 8 smooth rational curves of Dynkin type $4 A_{2}$, which was not known before.

Corollary 1.3 Let $X$ be a fake projective plane with $\operatorname{Aut}(X) \cong 7: 3$. Let $G=\operatorname{Aut}(X)$, $W=X / G$ and $v: V \rightarrow W$ be a minimal resolution. Then $W$ has 3 singular points of type $\frac{1}{3}(1,2)$ and 1 singular point of type $\frac{1}{7}(1,3)$. Furthermore, $V$ is a minimal elliptic surface of Kodaira dimension 1 with 2 multiple fibres and with 4 reducible fibres of type $I_{3}$. The pair of the multiplicities is the same as that of the minimal resolution of the order 7 quotient of $X$.

Corollary 1.4 Let $X$ be a fake projective plane with $\operatorname{Aut}(X) \cong 7: 3$. Let $G \cong \mathbb{Z} / 7 \mathbb{Z}<$ Aut $(X), Z=X / G$ and $v: Y \rightarrow Z$ be a minimal resolution. Then the elliptic fibration of $Y$ has 3 singular fibres of type $I_{1}$ and 1 reducible fibre of type $I_{9}$. 
Finally, we remark that there is not yet known a geometric construction of a fake projective plane that does not use the ball quotient construction. A main purpose of this paper is to provide some useful hints on how to find such a construction.

Acknowledgements I thank Gopal Prasad, Sai-Kee Yeung, Donald Cartwright for many helpful conversations, and especially Tim Steger for informing me of Lemma 4.3. I also thank Igor Dolgachev for his comments, which helped me to improve the exposition of the paper.

This research was supported by the Korea Research Foundation Grant(KRF-2007C00002).

Notation

$D_{1} \equiv D_{2}$ : two divisors $D_{1}$ and $D_{2}$ are linearly equivalent

$D_{1} \sim D_{2}:$ two $\mathbb{Q}$-divisors $D_{1}$ and $D_{2}$ are numerically equivalent

$K_{X}:$ the canonical divisor of $X$

$p_{g}(X)=\operatorname{dim} H^{2}\left(X, \mathcal{O}_{X}\right):$ the geometric genus of $X$

$q(X)=\operatorname{dim} H^{1}\left(X, \mathcal{O}_{X}\right):$ the irregularity of $X$

$\chi(X)=1-q(X)+p_{q}(X):$ the holomorphic Euler characteristic of $X$

$e(X)$ : the Euler number of $X$

$b_{i}(X)$ : the $i$-th Betti number of $X$

$c_{i}(X):$ the $i$-th Chern class of $X$

$c_{2}(X)=e(X)$ if $X$ is a smooth surface

$g(C)$ : the genus of a curve $C$

$(-n)$-curve : a smooth rational curve with self-intersection $-n$

singularity of type $\frac{1}{m}(1, a):$ a cyclic quotient singularity given by the diagonal action of $\operatorname{diag}\left(\zeta, \zeta^{a}\right)$ on $\mathbb{C}^{2}$, where $\zeta$ is a primitive $m$-th root of 1

$\mathbb{Q}$-homology $\mathbb{C P}^{2}$ : a normal projective surface with the same Betti numbers as $\mathbb{C} \mathbb{P}^{2}$

\section{Preliminary results}

There have been known many equivalent characterizations of a fake projective plane.

Theorem 2.1 A smooth compact complex surface $X$ with $b_{1}(X)=0, b_{2}(X)=1$ is a fake projective plane if one of the following holds true:

(1) $X$ is not isomorphic to $\mathbb{C} \mathbb{P}^{2}$. 
(2) $X$ is not homeomorphic to $\mathbb{C P}^{2}$.

(3) $X$ is not homotopy equivalent to $\mathbb{C P}^{2}$.

(4) $\pi_{1}(X)$ is an infinite group.

(5) The universal cover of $X$ is a 2-dimensional complex ball $B \subset \mathbb{C}^{2}$, and $X \cong$ $B / \pi_{1}(X)$, where $\pi_{1}(X) \subset \mathrm{PU}(2,1)$.

(6) $K_{X}$ is ample.

(7) $K_{X}$ is ample, $p_{g}(X)=q(X)=0$, and $K_{X}^{2}=3 c_{2}(X)=9$.

We need the following lemma.

Lemma 2.2 Let $X$ be a fake projective plane and $C$ be a smooth curve on $X$. Then $e(C) \leq-4$, or equivalently $g(C) \geq 3$.

Proof Let $l$ be an ample generator of $\operatorname{Pic}(X)$ modulo torsions. Then $l^{2}=1$ and $C \equiv \equiv_{\mathbb{Q}} m l$ for some positive integer $m$. Since $K_{X} \equiv_{\mathbb{Q}} 3 l$, we have

$$
e(C)=2-2 g(C)=-C^{2}-C K_{X}=-\left(m^{2}+3 m\right) \leq-4 .
$$

A normal projective complex surface is called a $\mathbb{Q}$-homology $\mathbb{C P} \mathbb{P}^{2}$ if it has the same Betti numbers with the complex projective plane $\mathbb{C} \mathbb{P}^{2}$. If a $\mathbb{Q}$-homology $\mathbb{C P}^{2}$ is nonsingular, then it is either $\mathbb{C P}^{2}$ or a fake projective plane.

Proposition 2.3 Let $S$ be a $\mathbb{Q}$-homology $\mathbb{C P}^{2}$ with quotient singularities only. Suppose that $S$ admits a finite group $G$ of automorphisms. Then the quotient $S / G$ is again a $\mathbb{Q}$-homology $\mathbb{C} \mathbb{P}^{2}$ with quotient singularities only.

In particular, $p_{g}(S / G)=q(S / G)=0, e(S / G)=3$ and $\chi(S / G)=1$.

Proof Since $S$ has $p_{g}=q=0$, so does the quotient $S / G$. Thus the minimal resolution of $S / G$ has $q=0$, and hence $b_{1}=0$. It follows that $b_{1}(S / G)=0$.

Since $S$ has $b_{2}=1$, so does the quotient $S / G$.

Now we consider fake projective planes with an automorphism, and get the following preliminary information.

Proposition 2.4 Let $X$ be a fake projective plane with an automorphism $\sigma$. Assume that the order of $\sigma$ is a prime number, say, $p$. Let $Z=X /\langle\sigma\rangle$ and $v: Y \rightarrow Z$ be a minimal resolution. Then the following holds: 
(1) $Z$ is a $\mathbb{Q}$-homology $\mathbb{C P}^{2}$ with $K_{Z}$ ample.

(2) $p_{g}(Y)=q(Y)=0$.

(3) $K_{Z}^{2}=9 / p$.

(4) The fixed point set $X^{\sigma}$ consists of 3 points.

Proof The statements (1) and (2) follow from Proposition 2.3, as $X$ itself is a $\mathbb{Q}-$ homology $\mathbb{C P}^{2}$ with $K_{X}$ ample.

Note that $Z$ is a $\mathbb{Q}$-factorial variety, $K_{Z}$ is a $\mathbb{Q}$-Cartier divisor, and

$$
K_{X} \sim \pi^{*} K_{Z}
$$

where $\pi: X \rightarrow Z$ is the quotient map.

Since $9=K_{X}^{2}=\left(\pi^{*} K_{Z}\right)^{2}=p K_{Z}^{2}$, (3) follows. Here and hereafter we use the intersection theory for $\mathbb{Q}$-divisors on $\mathbb{Q}$-factorial varieties (or, for topologists, on V-manifolds).

It remains to prove (4). Assume that $X^{\sigma}$ contains a curve. Since any two curves on a fake projective plane intersect, we may assume that $X^{\sigma}$ consists of a smooth curve $C$ and $r$ isolated points. Then the quotient surface $Z$ has $r$ singular points. Note that $e\left(X^{\sigma}\right)=r+e(C)$. Using Hurwitz formula, we have

$$
e(X)=p \cdot e(Z)-(p-1)\{r+e(C)\} .
$$

Since $e(X)=e(Z)=3$, this yields

$$
r+e(C)=3 .
$$

This also follows from the topological Lefschetz fixed point formula, as $\sigma$ acts identically on $H^{*}(X, \mathbb{Q})$.

From the orbifold Bogomolov-Miyaoka-Yau inequality, one sees that a $\mathbb{Q}$-homology $\mathbb{C P}^{2}$ with quotient singularities only cannot have more than 5 singular points (see eg Kollár [9] and Hwang and Keum [4]). Thus $r \leq 5$. This bound together with Lemma 2.2 contradicts $(2-1)$. Thus, $X^{\sigma}$ does not contain a curve and consists of 3 isolated points.

Corollary $2.5 p \neq 2$.

Proof Suppose $p=2$. Then $Z$ has rational double points only, hence $K_{Y} \sim v^{*} K_{Z}$ and $K_{Y}^{2}=K_{Z}^{2}=9 / 2$, which is not an integer, a contradiction. 
Prasad and Yeung [16] have provided precise possible values for the order $p$. According to their result, $p=3$ or 7 . In each case we will determine the types of singularities of the quotient surface $Z$, using the holomorphic Lefschetz fixed point formula.

Lemma 2.6 Let $S$ be a complex manifold of dimension 2 with $p_{g}=q=0$. Assume that $S$ admits an automorphism $\sigma$ of prime order $p$. Let $r_{i}(1 \leq i \leq p-1)$ be the number of isolated fixed points of $\sigma$ which give singularities of type $\frac{1}{p}(1, i)$ on the quotient surface. Let $C_{1}, \ldots, C_{k}$ be 1-dimensional components of the fixed locus $S^{\sigma}$. Then

where

$$
1=\sum_{j=1}^{k}\left\{\frac{1-g\left(C_{j}\right)}{2}+\frac{(p+1) C_{j}^{2}}{12}\right\}+\sum_{i=1}^{p-1} a_{i} r_{i}
$$

$$
a_{i}=\frac{1}{p-1} \sum_{j=1}^{p-1} \frac{1}{\left(1-\zeta^{j}\right)\left(1-\zeta^{i j}\right)}
$$

with $\zeta$ a primitive $p$-th root of 1 , eg $a_{1}=(5-p) / 12, a_{2}=(11-p) / 24$.

Proof This formula easily follows from the original holomorphic Lefschetz fixed point formula of Atiyah and Singer [1, p 567]. See, eg Zhang [17, Lemma 1.6], whose proof works for all complex manifolds of dimension 2 with $p_{g}=q=0$, not just for rational surfaces.

\section{The case $G$ contains a normal subgroup of order 3}

In this section we prove the following:

Proposition 3.1 Let $\sigma$ be an automorphism of order 3 of a fake projective plane $X$. Let $Z=X /\langle\sigma\rangle$ and $v: Y \rightarrow Z$ be a minimal resolution. Then $Z$ has 3 singularities of type $\frac{1}{3}(1,2)$, and $Y$ is a minimal surface of general type with $K_{Y}^{2}=3, p_{g}=0$.

Proof From Proposition 2.4, we see that $p_{g}(Y)=q(Y)=0$ and $K_{Z}^{2}=3$. Also we know that the fixed locus $X^{\sigma}$ consists of 3 points. Assume that $Z$ has $r_{i}$ singular points of type $\frac{1}{3}(1, i)$. By Lemma 2.6 we have

$$
1=a_{1} r_{1}+a_{2} r_{2}=\frac{1}{6} r_{1}+\frac{1}{3} r_{2} .
$$

Since $r_{1}+r_{2}=3$, we see that $r_{1}=0$ and $r_{2}=3$. This proves that $Z$ has 3 singular points of type $\frac{1}{3}(1,2)$. In particular, $K_{Y} \sim v^{*} K_{Z}$. Since $K_{Z}$ is ample, $K_{Y}$ is nef. Thus $Y$ is minimal. 
Corollary 3.2 Let $X$ be a fake projective plane with $\operatorname{Aut}(X) \cong(\mathbb{Z} / 3 \mathbb{Z})^{2}$. Let $G=$ Aut $(X), Z=X / G$ and $v: Y \rightarrow Z$ be a minimal resolution. Then $Z$ has 4 singular points of type $\frac{1}{3}(1,2)$, and $Y$ is a minimal surface of general type with $K_{Y}^{2}=1, p_{g}=0$.

Proof The group $G$ has 4 subgroups isomorphic to $\mathbb{Z} / 3 \mathbb{Z}$. Each fixes 3 isolated points of type $\frac{1}{3}(1,2)$. No stabilizer of a nonsingular point can be isomorphic to $(\mathbb{Z} / 3 \mathbb{Z})^{2}$, thus there are 12 points, each of whose stabilizers is isomorphic to $\mathbb{Z} / 3 \mathbb{Z}$. It follows that $Z$ has 4 singular points of type $\frac{1}{3}(1,2)$. Note that the canonical divisor $K_{Z}$ of $Z$ is $\mathbb{Q}$-Cartier and

$$
K_{X} \sim \pi^{*} K_{Z}
$$

where $\pi: X \rightarrow Z$ is the quotient map. Thus

$$
K_{Z}^{2}=K_{X}^{2} / 9=1 .
$$

Since $Z$ has only rational double points and $K_{Z}$ is ample, we see that $K_{Y}$ is nef, $K_{Y}^{2}=1$ and hence the assertion on $Y$ follows.

According to [16], many fake projective planes admit an automorphism of order 3, thus by taking a quotient one can obtain many new examples of a minimal surface of general type with $K_{Y}^{2}=3, p_{g}=0$.

Question Does there exist a fake projective plane $X$ with an automorphism $\sigma$ of order 3 such that the minimal resolution $Y$ of $Z=X /\langle\sigma\rangle$ is simply connected?

Remark 3.3 (1) Since $Z$ has rational singularities only, $\pi_{1}(Y) \cong \pi_{1}(Z)$. Thus the question is whether there is a fake projective plane $X$ with an automorphism $\sigma$ of order 3 such that the augmented fundamental group $\left\langle\pi_{1}(X), \tilde{\sigma}\right\rangle$, where $\widetilde{\sigma}$ is a lift of $\sigma$ to the ball, is the normal closure of the subgroup generated by elements of order 3 .

(2) In the case of order 7, there is a fake projective plane $X$ with an automorphism $\sigma$ of order 7 such that the minimal resolution $Y$ of the quotient is simply connected [8]. In this case $Y$ is not of general type.

(3) A simply connected surface of general type with $K^{2}=3, p_{g}=0$ has been recently constructed by H Park, J Park and D Shin [15]. They use the method of Lee and Park [11], which produces examples of simply connected surfaces of general type with $K^{2}=2, p_{g}=0$. An affirmative answer to the question would give yet another interesting example of a simply connected surface of general type with $K^{2}=3, p_{g}=0$. 


\section{The case $G$ contains a normal subgroup of order 7}

In this section we first prove the following:

Proposition 4.1 Let $\sigma$ be an automorphism of order 7 of a fake projective plane $X$. Let $Z=X /\langle\sigma\rangle$ and $v: Y \rightarrow Z$ be a minimal resolution. Then $Z$ has 3 singular points of type $\frac{1}{7}(1,3)$, and $K_{Y}^{2}=0$.

This follows from the following three lemmas.

Lemma 4.2 Let $\sigma$ be an automorphism of order 7 of a fake projective plane $X$. Let $Z=X /\langle\sigma\rangle$ and $v: Y \rightarrow Z$ be a minimal resolution. Then $Z$ has either 3 singular points of type $\frac{1}{7}(1,3)$, or 2 singular points of type $\frac{1}{7}(1,4)$, and 1 singular point of type $\frac{1}{7}(1,6)$.

Proof From Proposition 2.4, we know that the fixed locus $X^{\sigma}$ consists of 3 points. Assume that $Z$ has $r_{i}$ singular points of type $\frac{1}{7}(1, i)$. By Lemma 2.6 we have

$$
-r_{1}+r_{2}+2 r_{3}+r_{4}+2 r_{5}+4 r_{6}=6 .
$$

Adding this to $\sum r_{i}=3$, we get

$$
2\left(r_{2}+r_{4}\right)+3\left(r_{3}+r_{5}\right)+5 r_{6}=9 .
$$

If $r_{6}=0$, then $r_{3}+r_{5}=3$, hence we get 3 points of type $\frac{1}{7}(1,3)=\frac{1}{7}(1,5)$.

If $r_{6}=1$, then $r_{2}+r_{4}=2$, hence we get 1 point of type $\frac{1}{7}(1,6)$ and 2 points of type $\frac{1}{7}(1,2)=\frac{1}{7}(1,4)$. This proves the assertion.

Lemma 4.3 Let $\sigma$ be an automorphism of order 7 of a fake projective plane $X$. Then $\sigma$ cannot have a fixed point of type $\frac{1}{7}(1,4)$.

Proof Assume that $\sigma$ has a fixed point of type $\frac{1}{7}(1,4)$. Then the group acting on the complex ball $B \subset \mathbb{C}^{2}$ must contain a matrix $\in \mathrm{PU}(2,1)$ which diagonalizes as:

$$
M=\left(\begin{array}{ccc}
\alpha & 0 & 0 \\
0 & \alpha \zeta & 0 \\
0 & 0 & \alpha \zeta^{4}
\end{array}\right)
$$

where $\zeta=\zeta_{7}=e^{2 \pi i / 7}$ is the 7-th root of unity and $\alpha$ a complex number.

Using the notation of [16], we can choose this matrix to be in $\bar{\Gamma}$, which is contained in a rank 3 division algebra over the field denoted by $\ell$. Therefore

$$
\operatorname{tr}(M)=\alpha\left(1+\zeta+\zeta^{4}\right) \text { and } \operatorname{det}(M)=\alpha^{3} \zeta^{5}
$$


both must belong to $\ell$. Thus $\ell$ contains $\operatorname{tr}(M)^{3} / \operatorname{det}(M)$, which is equal to

$$
\left(1+\zeta+\zeta^{4}\right)^{3} / \zeta^{5}=6\left(\zeta+\zeta^{-1}\right)^{3}+\left(\zeta+\zeta^{-1}\right)^{2}-15\left(\zeta+\zeta^{-1}\right)+5 .
$$

The field which this generates over $\mathbb{Q}$, namely $\mathbb{Q}\left[\zeta+\zeta^{-1}\right]$, must be contained in $\ell$. None of the cases on Prasad-Yeung's final list has such an $\ell$. There is exactly one possibility listed, but later excluded, which does have such an $\ell$, namely $\mathcal{C}_{31}$.

Definition 4.4 When $Z$ has 3 singularities of type $\frac{1}{7}(1,3)$, we denote by

$$
A_{1}, A_{2}, A_{3}, B_{1}, B_{2}, B_{3}, C_{1}, C_{2}, C_{3}
$$

the exceptional curves of $v: Y \rightarrow Z$ whose Dynkin diagram is given by

$$
(-2)-(-2)-(-3) \quad(-2)-(-2)-(-3) \quad(-2)-(-2)-(-3)
$$

Lemma 4.5 Assume that $Z$ has 3 singularities of type $\frac{1}{7}(1,3)$. Then

$$
K_{Y} \sim v^{*} K_{Z}-\frac{1}{7}\left(A_{1}+2 A_{2}+3 A_{3}\right)-\frac{1}{7}\left(B_{1}+2 B_{2}+3 B_{3}\right)-\frac{1}{7}\left(C_{1}+2 C_{2}+3 C_{3}\right) .
$$

In particular, $K_{Y}^{2}=0$.

Proof This follows from the adjunction formula

$$
K_{Y} \sim v^{*} K_{Z}-D
$$

where $D$ is a $\mathbb{Q}$-linear combination of the exceptional curves $A_{i}, B_{i}, C_{i}$ with coefficients in the interval $[0,1)$. These coefficients can be uniquely determined by the system of 9 linear equations

$$
-2-E^{2}=K_{Y} \cdot E=-D \cdot E \text { for } \quad E=A_{i}, B_{i}, C_{i}(i=1,2,3) .
$$

Finally note that $K_{Z}^{2}=9 / 7$ by Proposition 2.4 . Also note that

$$
\left\{\frac{1}{7}\left(A_{1}+2 A_{2}+3 A_{3}\right)\right\}^{2}=-K_{Y} \cdot\left\{\frac{1}{7}\left(A_{1}+2 A_{2}+3 A_{3}\right)\right\}=-\frac{3}{7} .
$$

Hence the last assertion follows.

This completes the proof of Proposition 4.1. 
To complete the proof of Theorem 1.1, it suffices to prove the following:

Proposition 4.6 Assume that $Z$ has 3 singular points of type $\frac{1}{7}(1,3)$. Then there are three cases :

(i) $Y$ is a minimal elliptic surface of Kodaira dimension 1 with 2 multiple fibres with multiplicity 2 and 3 , respectively.

(ii) $Y$ is a minimal elliptic surface of Kodaira dimension 1 with 2 multiple fibres with multiplicity 2 and 4, respectively.

(iii) $Y$ is a minimal elliptic surface of Kodaira dimension 1 with 2 multiple fibres with multiplicity 3 and 3 , respectively.

The proof of Proposition 4.6 consists of several lemmas.

Lemma 4.7 Assume that $Z$ has 3 singularities of type $\frac{1}{7}(1,3)$.

(1) $-m K_{Y}$ is not effective for any positive integer $m$.

(2) The Kodaira dimension of $Y$ is at least 1.

Proof (1) Note that for $m \geq 1$

$$
\left(v^{*} K_{Z}\right) \cdot\left(-m K_{Y}\right)=-m\left(v^{*} K_{Z}\right)^{2}=\frac{-9 m}{7}<0 .
$$

Since $v^{*} K_{Z}$ is nef, $-m K_{Y}$ cannot be effective.

(2) By Proposition 2.4, $p_{g}(Y)=q(Y)=0$. By Proposition 4.1, $K_{Y}^{2}=0$. Thus if $Y$ is of Kodaira dimension $\leq 0$, then by the classification theory of surfaces $Y$ is either a rational surface or an Enriques surface.

From the Riemann-Roch theorem and (1) we see that for any integer $k \geq 2$

$$
h^{0}\left(k K_{Y}\right)=1+h^{1}\left(k K_{Y}\right) \geq 1 .
$$

Thus $Y$ is not rational.

Since $\left(v^{*} K_{Z}\right) \cdot\left(K_{Y}\right)=\left(v^{*} K_{Z}\right)^{2}=K_{Z}^{2}=9 / 7>0, K_{Y}$ is not numerically trivial, hence $Y$ is not an Enriques surface. This proves (2).

Note that $\operatorname{Pic}(Y) \cong H^{2}(Y, \mathbb{Z})$. Let $\operatorname{Pic}(Y)_{f}:=\operatorname{Pic}(Y) /$ torsion. With the intersection pairing $\operatorname{Pic}(Y)_{f}$ becomes a lattice. 
Lemma 4.8 Assume that $Z$ has 3 singularities of type $\frac{1}{7}(1,3)$. Then one can choose two $\mathbb{Q}$-divisors

$$
\begin{aligned}
L & =\frac{1}{7}\left(A_{1}+2 A_{2}+3 A_{3}\right)+\frac{2}{7}\left(B_{1}+2 B_{2}+3 B_{3}\right)+\frac{4}{7}\left(C_{1}+2 C_{2}+3 C_{3}\right), \\
M & =\frac{1}{3} v^{*} K_{Z}-\frac{2}{7}\left(B_{1}+2 B_{2}+3 B_{3}\right)+\frac{1}{7}\left(C_{1}+2 C_{2}+3 C_{3}\right),
\end{aligned}
$$

such that the lattice $\operatorname{Pic}(Y)_{f}$ is generated over the integers by the numerical equivalence classes of $M, L$ and the 8 curves $A_{2}, A_{3}, B_{1}, B_{2}, B_{3}, C_{1}, C_{2}, C_{3}$.

Proof Note first that in this case $K_{Y}^{2}=0$, hence by Noether formula $\operatorname{rank} \operatorname{Pic}(Y)_{f}=10$. Since $\operatorname{Pic}(Y)_{f}$ contains an element of self-intersection -3 , eg $A_{3}$, it is odd unimodular and of signature $(1,9)$.

Let $R$ be the sublattice of $\operatorname{Pic}(Y)_{f}$ generated by the numerical equivalence classes of the 9 curves $A_{1}, A_{2}, A_{3}, B_{1}, B_{2}, B_{3}, C_{1}, C_{2}, C_{3}$. Let $\bar{R}$ and $R^{\perp}$ be its primitive closure and its orthogonal complement, respectively, in the lattice $\operatorname{Pic}(Y)_{f}$. Note that $R^{\perp}$ is of rank 1 .

For an integral lattice $N$, let $\operatorname{disc}(N)$ denote the discriminant group of $N$

$$
\operatorname{disc}(N):=\operatorname{Hom}(N, \mathbb{Z}) / N .
$$

It is easy to see that $\operatorname{disc}(R) \cong(\mathbb{Z} / 7 \mathbb{Z})^{3}$, more precisely

$$
\operatorname{disc}(R)=\left\langle\frac{1}{7}\left(A_{1}+2 A_{2}+3 A_{3}\right), \frac{1}{7}\left(B_{1}+2 B_{2}+3 B_{3}\right), \frac{1}{7}\left(C_{1}+2 C_{2}+3 C_{3}\right)\right\rangle .
$$

Note that the length (the minimum number of generators) of $\operatorname{disc}(R)$ is 3 . Since the lattice $\operatorname{Pic}(Y)_{f}$ is unimodular, $\operatorname{disc}(\bar{R})$ is isomorphic to $\operatorname{disc}\left(R^{\perp}\right)$ which is of length 1. Hence $R$ must be of index 7 in $\bar{R}$, and the generator of $\bar{R} / R$ is of the form

$$
L=\frac{1}{7}\left(A_{1}+2 A_{2}+3 A_{3}\right)+\frac{a}{7}\left(B_{1}+2 B_{2}+3 B_{3}\right)+\frac{b}{7}\left(C_{1}+2 C_{2}+3 C_{3}\right) .
$$

Since both $L \cdot K_{Y}$ and $L^{2}$ must be integers, we see that $(a, b)=(2,4)$ or $(4,2)$ modulo 7. Thus up to interchanging the curves $B_{i}$ 's and $C_{i}$ 's, we have determined the divisor $L$ uniquely modulo $R$.

Now we have $\operatorname{disc}(\bar{R}) \cong \operatorname{disc}\left(R^{\perp}\right) \cong \mathbb{Z} / 7 \mathbb{Z}$. Note that the integral divisor $7 v^{*} K_{Z}$ belongs to $R^{\perp}$ and $\left(7 v^{*} K_{Z}\right)^{2}=7 \cdot 3^{2}$. Thus $R^{\perp}$ is generated by $\frac{7}{3} v^{*} K_{Z}$, hence

$$
\operatorname{disc}\left(R^{\perp}\right)=\left\langle\frac{1}{3} v^{*} K_{Z}\right\rangle
$$


On the other hand,

$$
\operatorname{disc}(\bar{R})=\langle L\rangle^{\perp} /\langle L\rangle=\left\langle\frac{3}{7}\left(B_{1}+2 B_{2}+3 B_{3}\right)+\frac{2}{7}\left(C_{1}+2 C_{2}+3 C_{3}\right)\right\rangle,
$$

where $\langle L\rangle=\bar{R} / R$ is the isotropic subgroup of $\operatorname{disc}(R)$ generated by $L(\bmod R)$ and $\langle L\rangle^{\perp}$ is its orthogonal complement in $\operatorname{disc}(R)$ with respect to the discriminant quadratic form on $\operatorname{disc}(R)$. (See, eg Nikulin [14] for discriminant quadratic forms for integral lattices.) Thus the index 7 extension $\bar{R} \oplus R^{\perp} \subset \operatorname{Pic}(Y)_{f}$ is given by the element of the form

$$
M=\frac{1}{3} v^{*} K_{Z}+a\left\{\frac{3}{7}\left(B_{1}+2 B_{2}+3 B_{3}\right)+\frac{2}{7}\left(C_{1}+2 C_{2}+3 C_{3}\right)\right\} .
$$

Since $M \cdot K_{Y}$ is an integer, we see that $a=4$ modulo 7. This determines the divisor $M$ uniquely modulo $R$.

Remark 4.9 The proof of Lemma 4.8 shows that the two $\mathbb{Q}$-divisors $M$ and $L$ are indeed integral divisors modulo torsion. The intersection matrix of the 10-divisors $M, L, A_{2}, A_{3}, B_{1}, B_{2}, B_{3}, C_{1}, C_{2}, C_{3}$ is given by:

$$
\left(\begin{array}{cccccccccc}
-2 & 0 & 0 & 0 & 0 & 0 & 2 & 0 & 0 & -1 \\
0 & -9 & 0 & -1 & 0 & 0 & -2 & 0 & 0 & -4 \\
0 & 0 & -2 & 1 & 0 & 0 & 0 & 0 & 0 & 0 \\
0 & -1 & 1 & -3 & 0 & 0 & 0 & 0 & 0 & 0 \\
0 & 0 & 0 & 0 & -2 & 1 & 0 & 0 & 0 & 0 \\
0 & 0 & 0 & 0 & 1 & -2 & 1 & 0 & 0 & 0 \\
2 & -2 & 0 & 0 & 0 & 1 & -3 & 0 & 0 & 0 \\
0 & 0 & 0 & 0 & 0 & 0 & 0 & -2 & 1 & 0 \\
0 & 0 & 0 & 0 & 0 & 0 & 0 & 1 & -2 & 1 \\
-1 & -4 & 0 & 0 & 0 & 0 & 0 & 0 & 1 & -3
\end{array}\right)
$$

It is easy to see that this matrix has determinant -1 . This double checks that our choice of $M, L$ and 8 curves was correct. But the unimodularity of the matrix only is not enough to prove Lemma 4.8, as it does not imply the integrality (modulo torsion) of $M$ and $L$. There are many possible choices of nonintegral $\mathbb{Q}$-divisors $M$ and $L$ making the matrix unimodular.

Lemma 4.10 Assume that $Z$ has 3 singularities of type $\frac{1}{7}(1,3)$. Then $Y$ does not contain a $(-1)$-curve (a smooth rational curve of self-intersection -1$) E$ with $0<E \cdot v^{*} K_{Z}<9 / 7$. 
Proof Assume that $Y$ contains such a $(-1)$-curve $E$. Write

$$
E \sim m M-d L+a_{2} A_{2}+a_{3} A_{3}+b_{1} B_{1}+b_{2} B_{2}+b_{3} B_{3}+c_{1} C_{1}+c_{2} C_{2}+c_{3} C_{3}
$$

with integer coefficients. Since $E \cdot v^{*} K_{Z}=m M \cdot v^{*} K_{Z}=3 m / 7$, the condition $0<E \cdot v^{*} K_{Z}<9 / 7$ is equivalent to $1 \leq m \leq 2$.

(1) Assume that $m=1$, ie

$$
E \sim M-d L+a_{2} A_{2}+a_{3} A_{3}+b_{1} B_{1}+b_{2} B_{2}+b_{3} B_{3}+c_{1} C_{1}+c_{2} C_{2}+c_{3} C_{3} .
$$

Then the coefficients in the above satisfy the following system of 9 inequalities and one equality:

$$
\begin{aligned}
& 0 \leq E \cdot A_{1}=a_{2} \\
& 0 \leq E \cdot A_{2}=-2 a_{2}+a_{3} \\
& 0 \leq E \cdot A_{3}=d+a_{2}-3 a_{3} \\
& 0 \leq E \cdot B_{1}=-2 b_{1}+b_{2} \\
& 0 \leq E \cdot B_{2}=b_{1}-2 b_{2}+b_{3} \\
& 0 \leq E \cdot B_{3}=2+2 d+b_{2}-3 b_{3} \\
& 0 \leq E \cdot C_{1}=-2 c_{1}+c_{2} \\
& 0 \leq E \cdot C_{2}=c_{1}-2 c_{2}+c_{3} \\
& 0 \leq E \cdot C_{3}=-1+4 d+c_{2}-3 c_{3} \\
& -1=E \cdot K_{Y}=-3 d+a_{3}+b_{3}+c_{3}
\end{aligned}
$$

From the 9 inequalities of the system, we obtain that

$$
a_{3} \leq \frac{2}{5} d, \quad b_{3} \leq \frac{3}{7}(2+2 d), \quad c_{3} \leq \frac{3}{7}(-1+4 d) .
$$

Indeed, eliminating $a_{2}$ from the second and the third inequality of the system, we get the first inequality of (4-1). Eliminating $b_{1}$ and $b_{2}$ from the fourth, the fifth and the sixth inequality of the system, we get the second inequality of (4-1). The third inequality of (4-1) can be proved similarly.

Also, we obtain the following bound for $d$ :

$$
0 \leq d \leq 50
$$

Indeed, from the first three inequalities of the system, we have

$$
d \geq-a_{2}+3 a_{3}=3\left(-2 a_{2}+a_{3}\right)+5 a_{2} \geq 5 a_{2} \geq 0 .
$$


Applying the three inequalities of (4-1) to the equality of the system, we get

$$
3 d-1=a_{3}+b_{3}+c_{3} \leq \frac{2}{5} d+\frac{3}{7}(2+2 d)+\frac{3}{7}(-1+4 d),
$$

hence $d \leq 50$.

We know that $E^{2}=-1$. Expanding $E^{2}$ using the intersection matrix from Remark 4.9 and then applying the equality of the system, we get

$$
\begin{aligned}
1+3 & d^{2}+2 d \\
& =(4+2 d) b_{3}+(6 d-2) c_{3}+\left(a_{2} A_{2}+a_{3} A_{3}\right)^{2}+\left(\sum_{i=1}^{3} b_{i} B_{i}\right)^{2}+\left(\sum_{i=1}^{3} c_{i} C_{i}\right)^{2} .
\end{aligned}
$$

Note that

$$
\begin{aligned}
\left(a_{2} A_{2}+a_{3} A_{3}\right)^{2} & =-2 a_{2}^{2}+2 a_{2} a_{3}-3 a_{3}^{2}=-2\left(a_{2}-\frac{1}{2} a_{3}\right)^{2}-\frac{5}{2} a_{3}^{2} \leq-\frac{5}{2} a_{3}^{2}, \\
\left(\sum_{i=1}^{3} b_{i} B_{i}\right)^{2} & =-2\left(b_{1}-\frac{1}{2} b_{2}\right)^{2}-\frac{3}{2}\left(b_{2}-\frac{2}{3} b_{3}\right)^{2}-\frac{7}{3} b_{3}^{2} \leq-\frac{7}{3} b_{3}^{2}, \\
\left(\sum_{i=1}^{3} c_{i} C_{i}\right)^{2} & =-2\left(c_{1}-\frac{1}{2} c_{2}\right)^{2}-\frac{3}{2}\left(c_{2}-\frac{2}{3} c_{3}\right)^{2}-\frac{7}{3} c_{3}^{2} \leq-\frac{7}{3} c_{3}^{2},
\end{aligned}
$$

forcing the above equality to give the following inequality:

$$
1+3 d^{2}+2 d \leq-\frac{5}{2} a_{3}^{2}-\frac{7}{3} b_{3}^{2}-\frac{7}{3} c_{3}^{2}+(4+2 d) b_{3}+(6 d-2) c_{3} .
$$

We claim that there is no integer solution satisfying (4-1)-(4-3) and the equality of the system, hence no solution satisfying the system.

The proof is cumbersome and goes as follows. First we obtain the list of solutions $\left(d, a_{3}, b_{3}, c_{3}\right)$ of the equality of the system under the constraints given by (4-2) and (4-1); for each value of $d$ from (4-2), we solve the equation $3 d-1=a_{3}+b_{3}+c_{3}$ in the range (4-1).

The following list is generated by a computer program.

$(40,16,35,68),(33,13,29,56),(30,12,26,51),(26,10,23,44),(25,10,22,42)$,

$(23,9,20,39),(20,8,18,33),(19,7,17,32),(18,7,16,30),(16,6,14,27)$,

$(15,6,13,25),(13,5,12,21),(12,4,11,20),(11,4,10,18),(10,4,9,16)$,

$(9,3,8,15),(8,3,7,13),(6,2,6,9),(5,2,5,7),(5,2,4,8),(5,1,5,8)$,

$(4,1,4,6),(3,1,3,4),(2,0,2,3),(1,0,1,1),(0,0,0,-1)$ 
Next, it is easy to check that none of these satisfies (4-3).

(2) Assume that $m=2$, ie

$$
E \sim 2 M-d L+a_{2} A_{2}+a_{3} A_{3}+b_{1} B_{1}+b_{2} B_{2}+b_{3} B_{3}+c_{1} C_{1}+c_{2} C_{2}+c_{3} C_{3} .
$$

In this case the coefficients satisfy the following system of 9 inequalities and one equality:

$$
\begin{aligned}
& 0 \leq E \cdot A_{1}=a_{2} \\
& 0 \leq E \cdot A_{2}=-2 a_{2}+a_{3} \\
& 0 \leq E \cdot A_{3}=d+a_{2}-3 a_{3} \\
& 0 \leq E \cdot B_{1}=-2 b_{1}+b_{2} \\
& 0 \leq E \cdot B_{2}=b_{1}-2 b_{2}+b_{3} \\
& 0 \leq E \cdot B_{3}=4+2 d+b_{2}-3 b_{3} \\
& 0 \leq E \cdot C_{1}=-2 c_{1}+c_{2} \\
& 0 \leq E \cdot C_{2}=c_{1}-2 c_{2}+c_{3} \\
& 0 \leq E \cdot C_{3}=-2+4 d+c_{2}-3 c_{3} \\
& -1=E \cdot K_{Y}=-3 d+a_{3}+b_{3}+c_{3}
\end{aligned}
$$

Also in this case, (4-1)-(4-3) are replaced by

$$
\begin{aligned}
& a_{3} \leq \frac{2}{5} d, \quad b_{3} \leq \frac{3}{7}(4+2 d), \quad c_{3} \leq \frac{3}{7}(-2+4 d) . \\
& 0 \leq d \leq 65 . \\
& 7+3 d^{2}+2 d \leq-\frac{5}{2} a_{3}^{2}-\frac{7}{3} b_{3}^{2}-\frac{7}{3} c_{3}^{2}+(8+2 d) b_{3}+(6 d-4) c_{3} .
\end{aligned}
$$

The same argument as in the case $m=1$ shows that there is no solution satisfying (4-4)-(4-6) and the equality of the system, hence no solution satisfying the system.

Lemma 4.11 Assume that $Z$ has 3 singularities of type $\frac{1}{7}(1,3)$. Then $Y$ is minimal.

Proof From Proposition 4.1 we know that $K_{Y}^{2}=0$.

Suppose $Y$ is not minimal. Then by Lemma 4.7 (2), $Y$ is of general type. Let $\mu: Y \rightarrow Y^{\prime}$ be a birational morphism to the minimal model $Y^{\prime}$. Then

$$
K_{Y} \equiv \mu^{*} K_{Y^{\prime}}+\sum E_{i}
$$


where $E_{i}$ 's are effective divisors, not necessarily irreducible, with $E_{i}^{2}=-1, E_{i} \cdot E_{j}=0$ for $i \neq j$. Note that a positive multiple of $\mu^{*} K_{Y^{\prime}} \equiv K_{Y}-\sum E_{i}$ is effective. Since $v^{*} K_{Z}$ is nef, we have

$$
\left(K_{Y}-\sum E_{i}\right) \cdot v^{*} K_{Z} \geq 0 .
$$

Furthermore, $\mu^{*} K_{Y^{\prime}}$ has positive self-intersection, thus by Hodge index theorem

$$
\left(K_{Y}-\sum E_{i}\right) \cdot v^{*} K_{Z} \neq 0 .
$$

Summarizing these, we have

$$
\left(K_{Y}-\sum E_{i}\right) \cdot v^{*} K_{Z}>0 .
$$

Let $E$ be a $(-1)$-curve on $Y$. Since $v^{*} K_{Z}$ is nef and $E$ is not contracted by $v$, we have

$$
E \cdot v^{*} K_{Z}>0
$$

On the other hand, by (4-7) we have

$$
E \cdot v^{*} K_{Z}<K_{Y} \cdot v^{*} K_{Z}=9 / 7 \text {. }
$$

Thus, the assertion follows from Lemma 4.10.

Proof of Proposition 4.6 By Lemma 4.7 and Lemma 4.11, $Y$ is a minimal elliptic surface of Kodaira dimension 1. It remains to prove the assertion on multiplicities of multiple fibres.

Let $|F|$ be the elliptic pencil on $Y$. By the canonical bundle formula for elliptic fibrations (see eg Barth et al [2, Chapter V]),

$$
F \sim n K_{Y}
$$

for some positive rational number $n$. We claim that $n$ must be an integer. To see this, we first note that $Y$ contains a (-3)-curve, eg the curve $A_{3}$ (Definition 4.4), hence $A_{3} \cdot K_{Y}=1$. Thus $n=A_{3} \cdot F$ is an integer.

Let $m_{1} F_{1}, m_{2} F_{2}, \ldots, m_{r} F_{r}$ be the multiple fibres of the elliptic fibration with multiplicity $m_{1}, m_{2}, \ldots, m_{r}$, respectively. Since $Y$ is not rational, $r \geq 2$. Again by the canonical bundle formula for elliptic fibrations,

$$
K_{Y} \equiv-F+\sum_{i=1}^{r}\left(m_{i}-1\right) F_{i} \equiv(r-1) F-\sum_{i=1}^{r} F_{i},
$$


hence

$$
\frac{1}{n}=r-1-\sum_{i=1}^{r} \frac{1}{m_{i}} .
$$

Since $\sum_{i=1}^{r} 1 / m_{i} \leq r / 2$, (4-8) implies that $r \leq 3$ if $n=2 ; r=2$ if $n \geq 3$. Since $n=A_{3} \cdot F=m_{i} A_{3} \cdot F_{i}$, all $m_{i}$ divide $n$. With these, a further analysis of (4-8) shows that if $n=2$, then $\left(m_{1}, m_{2}, m_{3}\right)=(2,2,2)$; if $n=3$, then $\left(m_{1}, m_{2}\right)=(3,3)$; if $n=4$, then $\left(m_{1}, m_{2}\right)=(2,4)$; if $n=6$, then $\left(m_{1}, m_{2}\right)=(2,3)$; if $n=5$ or $n \geq 7$, then there is no solution for $m_{i}$ 's. The first case would imply that there is a degree 2 map : $A_{3} \rightarrow \mathbb{P}^{1}$, ramified at 3 points, which is impossible. This completes the proof of Proposition 4.6.

Corollary 4.12 Let $X$ be a fake projective plane with $\operatorname{Aut}(X) \cong 7: 3$. Let $G=$ Aut $(X), W=X / G$ and $v: V \rightarrow W$ be a minimal resolution. Then $W$ has 3 singular points of type $\frac{1}{3}(1,2)$ and 1 singular point of type $\frac{1}{7}(1,3)$. Furthermore, $V$ is a minimal elliptic surface of Kodaira dimension 1 with 2 multiple fibres and with 4 reducible fibres of type $I_{3}$. The pair of the multiplicities is the same as that of the minimal resolution of the order 7 quotient of $X$.

\section{Proof Write}

$$
G=\left\langle\sigma, \tau \mid \sigma^{7}=\tau^{3}=1, \tau \sigma \tau^{-1}=\sigma^{2}\right\rangle
$$

Let $Z=X /\langle\sigma\rangle$ be the order 7 quotient of $X$ and $Y$ be a minimal resolution of $Z$. Then by Proposition 4.6, $Z$ has Kodaira dimension 1 and has 3 singular points of type $\frac{1}{7}(1,3)$, which form a single orbit of the induced automorphism $\bar{\tau}$. By Proposition 3.1 , every element of order 3 of $G$ fixes 3 points of type $\frac{1}{3}(1,2)$. No stabilizer of a nonsingular point can be isomorphic to $7: 3$, thus $W=Z /\langle\bar{\tau}\rangle$ has 3 singular points of type $\frac{1}{3}(1,2)$ and 1 singular point of type $\frac{1}{7}(1,3)$.

Note that the canonical divisor $K_{W}$ of $W$ is an ample $\mathbb{Q}$-Cartier divisor and

$$
K_{W}^{2}=\frac{K_{X}^{2}}{21}=\frac{3}{7}
$$

Thus by adjunction formula $K_{V}^{2}=0$. Since $Y$ has Kodaira dimension $1, V$ has Kodaira dimension $\leq 1$. Note that the action of $\bar{\tau}$ on $Z$ lifts to $Y$. Let $W^{\prime}=Y /\langle\bar{\tau}\rangle$. By Proposition 4.6, we know that $K_{Y}$ is nef. Thus $K_{W^{\prime}}$ is nef. Since $W^{\prime}$ has 3 singular points of type $\frac{1}{3}(1,2)$, and since $V$ is the minimal resolution of $W^{\prime}$, we see that $K_{V}$ is nef. This proves that $V$ is minimal and of Kodaira dimension $\geq 0$. Note that

$$
\left(v^{*} K_{W}\right) \cdot\left(K_{V}\right)=K_{W}^{2}=\frac{3}{7}>0,
$$


thus $K_{V}$ is not numerically trivial. This proves that $V$ has Kodaira dimension 1 . The elliptic fibration on $V$ is given by a multiple of $K_{V}$.

Now $V$ has 9 smooth rational curves coming from the resolution $v: V \rightarrow W$. The eight $(-2)$-curves among them must be contained in fibres of the elliptic fibration. This is possible only if the fibres are the union of 4 reducible fibres of type $I_{3}$, since $V$ has Picard number 10.

Note that $Y$ is the degree 3 cover of $W^{\prime}$ branched along the 3 singular points of $W^{\prime}$, and $W^{\prime}$ has an elliptic fibration structure with a $(-3)$-curve that is a multisection. The (-3)-curve on $W^{\prime}$ splits in $Y$ giving three $(-3)$-curves, thus the elliptic fibres of $W^{\prime}$ do not split in $Y$. The fibre containing one of the singular point of $W^{\prime}$ gives a fibre of type $I_{1}$, the fibre of type $I_{3}$ gives a fibre of type $I_{9}$, and the multiple fibres give multiple fibres of the same multiplicities.

Remark 4.13 (1) By Proposition 4.6, the elliptic fibration on $V$ has multiplicities $(2,3)$ or $(2,4)$ or $(3,3)$. Such an elliptic surface with multiplicities $(2,3)$ was constructed by Ishida [5]. His construction was based on the description of Mumford surface as a ball quotient. From his elliptic surface we constructed a fake projective plane [8].

(2) By Cartwright and Steger such an elliptic surface with multiplicities $(2,4)$ exists. From such an elliptic surface one can give a similar construction of a fake projective plane [7].

From the proof of Corollary 4.12, we also have the following:

Corollary 4.14 Let $X$ be a fake projective plane with $\operatorname{Aut}(X) \cong 7: 3$. Let $G \cong$ $\mathbb{Z} / 7 \mathbb{Z}<\operatorname{Aut}(X), Z=X / G$ and $v: Y \rightarrow Z$ be a minimal resolution. Then the elliptic fibration of $Y$ has 3 singular fibres of type $I_{1}$ and 1 reducible fibre of type $I_{9}$.

\section{References}

[1] MF Atiyah, I M Singer, The index of elliptic operators. III, Ann. of Math. (2) 87 (1968) 546-604 MR0236952

[2] W P Barth, K Hulek, C A M Peters, A Van de Ven, Compact complex surfaces, second edition, Ergebnisse 3rd Series: Modern Surveys in Mathematics 4, Springer, Berlin (2004) MR2030225

[3] I Dolgachev, Algebraic surfaces with $q=p_{g}=0$, from: "CIME Algebraic surfaces", Liguori, Naples (1981) 97-216

[4] D Hwang, J Keum, The maximum number of singular points on rational homology projective planes arXiv:math.AG/0801.3021 
[5] M-N Ishida, An elliptic surface covered by Mumford's fake projective plane, Tohoku Math. J. (2) 40 (1988) 367-396 MR957050

[6] M-N Ishida, F Kato, The strong rigidity theorem for non-Archimedean uniformization, Tohoku Math. J. (2) 50 (1998) 537-555 MR1653430

[7] J Keum, A fake projective plane constructed from an elliptic surface with multiplicities $(2,4)$, Preprint

[8] J Keum, A fake projective plane with an order 7 automorphism, Topology 45 (2006) 919-927 MR2239523

[9] J Kollár, Is there a topological Bogomolov-Miyaoka-Yau inequality?, Pure Appl. Math. Q. 4 (2008) 203-236 MR2400877

[10] A Kurihara, Construction of p-adic unit balls and the Hirzebruch proportionality, Amer. J. Math. 102 (1980) 565-648 MR573103

[11] Y Lee, J Park, A simply connected surface of general type with $p_{g}=0$ and $K^{2}=2$, Invent. Math. 170 (2007) 483-505 MR2357500

[12] D Mumford, An algebraic surface with $K$ ample, $\left(K^{2}\right)=9, p_{g}=q=0$, Amer. J. Math. 101 (1979) 233-244 MR527834

[13] G A Mustafin, Non-Archimedean uniformization, Uspehi Mat. Nauk 33 (1978) 225226 MR0476763

[14] V V Nikulin, Integer symmetric bilinear forms and some of their geometric applications, Izv. Akad. Nauk SSSR Ser. Mat. 43 (1979) 111-177, 238 MR525944

[15] H Park, J Park, D Shin, A simply connected surface of general type with $p_{g}=0$ and $K^{2}=3$ arXiv:math.AG/07080273

[16] G Prasad, S-K Yeung, Fake projective planes, Invent. Math. 168 (2007) 321-370 MR2289867

[17] D-Q Zhang, Automorphisms of finite order on rational surfaces, J. Algebra 238 (2001) 560-589 MR1823774 With an appendix by I Dolgachev

School of Mathematics, Korea Institute for Advanced Study

Seoul 130-722, Korea

jhkeum@kias.re.kr

Proposed: Ron Stern

Seconded: Ron Fintushel, Walter Neumann

Received: 3 March 2008

Revised: 14 June 2008 